\title{
A WEIGHTED WEAK TYPE INEQUALITY FOR THE MAXIMAL FUNCTION
}

\author{
E. SAWYER
}

\begin{abstract}
We show that the operator $S=v^{-1} M v$, where $M$ denotes the HardyLittlewood maximal operator, is of weak type $(1,1)$ with respect to the measure $v(x) w(x) d x$ whenever $v$ and $w$ are $A_{1}$ weights. B. Muckenhoupt's weighted norm inequality for the maximal function can then be obtained directly from the P. Jones factorization of $A_{p}$ weights using interpolation with change of measure.
\end{abstract}

In [8], B. Muckenhoupt characterized the nonnegative functions, or weights, $w$ on $R$ satisfying the weighted norm inequality $(1<p<\infty)$

$$
\int_{-\infty}^{\infty}|M f(x)|^{p} w(x) d x \leqslant C \int_{-\infty}^{\infty}|f(x)|^{p} w(x) d x \quad \text { for all } f
$$

where $M f(x)=\left(\sup _{x \in I} 1 /|I|\right) \int_{I}|f(y)| d y$ is the Hardy-Littlewood maximal function of $f$, as those weights $w$ satisfying the $A_{p}$ condition

$\left(A_{p}\right) \quad\left[\frac{1}{|I|} \int_{I} w\right]\left[\frac{1}{|I|} \int_{I} w^{1-p^{\prime}}\right]^{p-1} \leqslant C$ for all intervals $I$.

Later, P. Jones showed in [7] that a weight $w$ satisfies the $A_{p}$ condition if and only if it admits a factorization

$$
w=w_{0} w_{1}^{1-p} \text { where } M w_{j}(x) \leqslant C w_{j}(x) \text { for all } x, j=0,1 .
$$

More recently, M. Christ and R. Fefferman [2] have given an elementary proof of the implication $\left(A_{p}\right) \Rightarrow(1)$ (see also R. Hunt, D. Kurtz and C. Neugebauer [5], B. Jawerth [6] and E. Sawyer [10]), and R. Coifman, P. Jones and J. Rubio de Francia [4] have given a short proof that (2) follows from (1) and its "dual" inequality, the boundedness of $M$ on $L^{p^{\prime}}\left(w^{1-p^{\prime}}\right)$.

A natural approach to obtaining inequality (1) directly from the factorization in (2) is provided by the Stein-Weiss interpolation with change of measures theorem [11]. In order to see what is needed, suppose (2) holds and, following the proof in [11], define $S f=w_{1}^{-1} M\left(w_{1} f\right)$. Note that (1) can be rewritten

$$
\int|S f|^{p} w_{0} w_{1} \leqslant C \int|f|^{p} w_{0} w_{1} \text { for all } f .
$$

Received by the editors October 18, 1983.

1980 Mathematics Subject Classification. Primary 42B25.

(c1985 American Mathematical Society $0002-9939 / 85 \$ 1.00+\$ .25$ per page 
Now $S$ is bounded on $L^{\infty}\left(w_{0} w_{1}\right)$ simply because $M w_{1} \leq C w_{1}$ and thus (3) will follow from the usual Marcinkiewicz interpolation theorem provided that $S$ is of weak type $(1,1)$ with respect to the measure $w_{0}(x) w_{1}(x) d x$.

Theorem. Suppose $v$ and $w$ satisfy the $A_{1}$ condition, i.e. $M v(x) \leqslant A v(x)$ and $M w(x) \leqslant B w(x)$ for all $x$. Then

$$
\int_{\left\{M g>v^{0}\right\}} v(x) w(x) d x \leqslant C \int_{-\infty}^{\infty} g(x) w(x) d x \text { for all } g \geqslant 0 \text { on } R,
$$

where the constant $C$ depends only on $A$ and $B$. This shows (with $g=\lambda^{-1} f v$ ) that the operator $S f=v^{-1} M(v f)$ is of weak type $(1,1)$ with respect to $v(x) w(x) d x$.

It would be of interest to obtain an analogue of the above approach for two weight inequalities and other operators, specifically the Hilbert transform. Regarding earlier work and other weighted weak type inequalities for the maximal function, see K. Andersen and B. Muckenhoupt [1], B. Muckenhoupt [8] and especially the treatment given by B. Muckenhoupt and R. L. Wheeden in [9] from which our proof borrows heavily. The letter $C$ will denote a positive constant that may change from line to line and $|E|_{v}=\int_{E} v(x) d x,|E|=\int_{E} d x$ for $v \geqslant 0$ on $R, E \subset R$.

Proof. It suffices to prove (4) for $g \geqslant 0$ bounded with compact support. Fix such a $g$. For $k \in Z$, let $\left\{I_{j}^{k}\right\}_{j}$ be the collection of component intervals of the open set $\Omega_{k}=\left\{M v>3^{k}\right\} \cap\left\{M g>3^{k}\right\}$. Denote by $\Gamma$ the set of pairs $(k, j)$ such that $I_{j}^{k} \cap\left\{v \leqslant 3^{k+1}\right\}$ has positive measure. For $(k, j) \in \Gamma$ we then have

$$
\begin{aligned}
& \frac{3^{k}}{A} \leqslant A^{-1} \underset{I_{j}^{k}}{\operatorname{essinf}} M v \leqslant \underset{I_{j}^{k}}{\operatorname{essinf}} v \leqslant\left|I_{j}^{k}\right|^{-1} \int_{I_{j}^{k}} v \\
& \leqslant \underset{I_{j}^{k}}{\operatorname{ess} \inf } M v \leqslant A \underset{I_{j}^{k}}{\operatorname{essinf}} v \leqslant 3^{k+1} A,
\end{aligned}
$$

since $M v \leqslant A v$. Thus

$$
\begin{aligned}
\int_{\left\{M g>v^{\prime}\right\}} v w & \leqslant 3 \sum_{k} 3^{k}\left|\left\{3^{k}<v \leqslant 3^{k+1}\right\} \cap\{M g>v\}\right|_{w} \\
& \leqslant 3 \sum_{k} 3^{k} \sum_{j:(k, j) \in \Gamma}\left|I_{j}^{k}\right|_{w} \\
& \leqslant\left. 3 A \sum_{(k, j) \in \Gamma}\left|I_{j}^{k}\right|^{-1}\left|I_{j}^{k}\right|_{v} I_{j}^{k}\right|_{w} \text { by (5). }
\end{aligned}
$$

For $N \in Z$, set $\Gamma_{N}=\{(k, j) \in \Gamma: k \geqslant N\}$. We shall prove

$$
\sum_{(k, j) \in \Gamma_{N}}\left|I_{j}^{k}\right|^{-1}\left|I_{j}^{k}\right|_{v}\left|I_{j}^{k}\right|_{w} \leqslant C \int g w \text { for all } N
$$

with a constant $C$ independent of $N$. The proof uses a variant of an idea of B. Muckenhoupt and R. L. Wheeden [9; Proof of Lemma 2]. First note that for $(k, j)$, $(t, s) \in \Gamma_{N}$ with $k \geqslant t$, either $I_{j}^{k} \subset I_{s}^{t}$ or $I_{j}^{k} \cap I_{s}^{t}=\varnothing$. Fix $N$ and let $G_{0}$ consist of the indices $(k, j) \in \Gamma_{N}$ for which $I_{j}^{k}$ is maximal in $\left\{I_{s}^{t}:(t, s) \in \Gamma_{N}\right\}$. Since $M v \leqslant A v$, $v$ satisfies the $A_{\infty}$ condition [3] and thus there are positive constants $C, \varepsilon$ such that

$$
|E|_{v} /|I|_{v} \leqslant C(|E| /|I|)^{\varepsilon} \quad \text { whenever } E \text { is a subset of an interval } I \text {. }
$$


Choose $0<\delta<\varepsilon$. If $G_{n}$ has been defined, let $G_{n+1}$ consist of those $(k, j) \in \Gamma$ for which there is $(t, s) \in G_{n}$ with $I_{j}^{k} \subset I_{s}^{t}$ and

(i) $\frac{1}{\left|I_{j}^{k}\right|} \int_{I_{j}^{k}} w>3^{(k-t) \delta} \frac{1}{\left|I_{s}^{t}\right|} \int_{I_{s}^{\prime}} w$,

(ii) $\frac{1}{\left|I_{i}^{l}\right|} \int_{I_{i}^{\prime}} w \leqslant 3^{(l-t) \delta} \frac{1}{\left|I_{s}^{t}\right|} \int_{I_{s}^{\prime}} w \quad$ whenever $(l, i) \in \Gamma$ and $I_{j}^{k} \subsetneq I_{i}^{l} \subset I_{s}^{t}$.

Let $P=\bigcup_{n=0}^{\infty} G_{n}$. Following [9], we claim that

$$
\sum_{(k, j) \in \Gamma_{N}}\left|I_{j}^{k}\right|^{-1}\left|I_{j}^{k}\right|_{v}\left|I_{j}^{k}\right|_{w} \leqslant C \sum_{(k, j) \in P}\left|I_{j}^{k}\right|^{-1}\left|I_{j}^{k}\right|_{v}\left|I_{j}^{k}\right|_{w} .
$$

To see this, suppose $(t, s) \in P$ and let $Q=Q(t, s)$ denote the set of indices $(k, j) \in \Gamma$ such that $I_{j}^{k} \subset I_{s}^{t}$ and there is no $(l, i) \in P$ with $I_{j}^{k} \subset I_{i}^{l} \subsetneq I_{s}^{t}$. Then by (9)(ii)

$$
\begin{aligned}
\sum_{(k, j) \in Q}\left|I_{j}^{k}\right|^{-1}\left|I_{j}^{k}\right|_{w}\left|I_{j}^{k}\right|_{v} & \leqslant \sum_{(k, j) \in Q} 3^{(k-t) \delta}\left|I_{s}^{t}\right|^{-1}\left|I_{s}^{t}\right|_{w}\left|I_{j}^{k}\right|_{v} \\
& \leqslant\left|I_{s}^{t}\right|^{-1}\left|I_{s}^{t}\right|_{w} \sum_{k=t}^{\infty} 3^{(k-t) \delta}\left|I_{s}^{t}\right|_{v} C\left(\frac{\left|\left\{M v>3^{k}\right\} \cap I_{s}^{t}\right|}{\left|I_{s}^{t}\right|}\right)^{\varepsilon} \text { by (8). }
\end{aligned}
$$

However, $M v \leqslant A v$ and so $\left|\left\{M v>3^{k}\right\} \cap I_{s}^{t}\right| \leqslant A 3^{-k}\left|I_{s}^{t}\right|_{v} \leqslant A^{2} 3^{t-k+1}\left|I_{s}^{t}\right|$ by (5) and thus the final line above is dominated by

$$
\begin{aligned}
C A^{2 \varepsilon}\left|I_{s}^{t}\right|^{-1}\left|I_{s}\right|_{w}\left|I_{s}^{t}\right|_{v} \sum_{k=t}^{\infty} 3^{(k-t) \delta} 3^{(t-k+1) \varepsilon} \\
\quad \leqslant C\left|I_{s}^{t}\right|^{-1}\left|I_{s}^{t}\right|_{w}\left|I_{s}^{t}\right|_{v} \quad \text { since } 0<\delta<\varepsilon .
\end{aligned}
$$

Inequality (10) now follows since $\bigcup_{(t, s) \in P} Q(t, s)=\Gamma_{N}$.

For each $k$ in $Z$, let $\left\{J_{i}^{k}\right\}_{i}$ be the component intervals of $\left\{M g>3^{k}\right\}$. Then $M\left(\chi_{J_{i}^{k}} g\right)>3^{k}$ on $J_{i}^{k}$ and so

$$
\left|J_{i}^{k}\right| \leqslant\left|\left\{M\left(\chi_{J_{i}^{k}} g\right)>3^{k}\right\}\right| \leqslant C 3^{-k} \int_{J_{i}^{k}} g
$$

since the maximal operator is of weak type $(1,1)$ with respect to Lebesgue measure. Given an interval $I_{j}^{k}$, there is a unique $i=i(k, j)$ such that $I_{j}^{k} \subset J_{i}^{k}$. From now on, whenever the index $i$ appears in a summation over $(k, j)$, it is understood that $i=i(k, j)$. We have

$$
\begin{aligned}
\sum_{(k, j) \in P}\left|I_{j}^{k}\right|^{-1}\left|I_{j}^{k}\right|_{v}\left|I_{j}^{k}\right|_{w} & \leqslant 3 A \sum_{(k, j) \in P} 3^{k}\left|I_{j}^{k}\right|_{w} \text { by }(5) \\
& \leqslant C A \sum_{(k, j) \in P} \frac{1}{\left|J_{i}^{k}\right|}\left(\int_{J_{i}^{k}} g\right)\left|I_{j}^{k}\right|_{w} \text { by (11) } \\
& =C A \int\left[\sum_{(k, j) \in P}\left|J_{i}^{k}\right|^{-1}\left|I_{j}^{k}\right|_{w} \chi_{J_{i}^{k}}\right] g .
\end{aligned}
$$


Let $h(x)=\sum_{(k, j) \in P}\left|J_{i}^{k}\right|^{-1}\left|I_{j}^{k}\right|_{w} \chi_{J_{i}^{k}}(x)$. It remains to show that $h(x) \leqslant C w(x)$ for all $x \in R$. So fix $x \in R$. For any given $k$, there is at most one interval $J_{i}^{k}$ containing $x$. We denote this interval, when it exists, by $J^{k}$. Let $P_{k}=\left\{(k, j) \in P: I_{j}^{k} \subset J^{k}\right\}$ and let $G=\left\{k: P_{k} \neq \varnothing\right\}$. Let $k_{0}$ be the least integer $k$ in $G$ and if $k_{0}, k_{1}, \ldots, k_{n}$ have been defined, choose $k_{n+1}$ in $G$ such that $k_{n+1}>k_{n}$ and

$$
\begin{aligned}
& \text { (i) } \frac{1}{\mid J^{k_{n+1} \mid}} \int_{J^{k_{n+1}}} w>2 \frac{1}{\mid J^{k_{n} \mid}} \int_{J^{k_{n}}} w, \\
& \text { (ii) } \frac{1}{\left|J^{l}\right|} \int_{J^{\prime}} w \leqslant 2 \frac{1}{\left|J^{k_{n}}\right|} \int_{J^{k_{n}}} w \text { for } k_{n} \leqslant l<k_{n+1}, l \in G .
\end{aligned}
$$

We now claim that

$$
\sum_{\substack{l \in G \\ k_{n} \leqslant l<k_{n+1}}} \sum_{(I, j) \in P_{l}} \frac{\left|I_{j}^{l}\right|_{w}}{\left|J^{l}\right|_{w}} \leqslant C \text { for } n \geqslant 0 .
$$

First, we observe that if $(l, j) \in P_{l}, k_{n} \leqslant l<k_{n+1}$, then

$$
\frac{1}{\left|I_{j}^{l}\right|} \int_{I_{j}^{\prime}} w>\frac{3^{\left(l-k_{n}\right) \delta}}{2 B} \frac{1}{\left|J^{l}\right|} \int_{J^{l}} w .
$$

To see this, let $I_{s}^{k_{n}}$ be the component of $\Omega_{k_{n}}$ that contains $I_{j}^{l}$. We claim that $\left(k_{n}, s\right) \in \Gamma$. Since $P \subset \Gamma$, it suffices to consider the case $\left(k_{n}, s\right) \notin P$. Since $k_{n} \in G$, $J^{k_{n}}$ must contain at least one interval of the form $I_{u}^{k_{n}}$ with $\left(k_{n}, u\right) \in P$ and it follows that $J^{k_{n}} \supsetneq I_{s}^{k_{n}}$. By the definition of $\Omega_{k_{n}}$, we must have $M v \leqslant 3^{k_{n}}$ at one of the endpoints of $I_{s}^{k_{n}}$. Thus the average of $v$ over $I_{s}^{k_{n}}$ is at most $3^{k_{n}}$ and so

$$
\left|\left\{v \leqslant 3^{k_{n}+1}\right\} \cap I_{s}^{k_{n}}\right|>0 .
$$

Hence $\left(k_{n}, s\right) \in \Gamma$ by definition. Now let $I_{\sigma}^{k}$ denote the smallest interval containing $I_{s}^{k_{n}}$ with $(k, \sigma) \in P$. Sufficiently many applications of (9)(i) yield

$$
\frac{1}{\left|I_{j}^{l}\right|} \int_{I_{j}^{l}} w>3^{(l-k) \delta} \frac{1}{\left|I_{\sigma}^{k}\right|} \int_{I_{\sigma}^{k}} w
$$

and, since $\left(k_{n}, s\right) \in \Gamma$, (9)(ii) shows that

$$
\frac{1}{\mid I_{s}^{k_{n} \mid}} \int_{I_{s}^{k_{n}}} w \leqslant 3^{\left(k_{n}-k\right) \delta} \frac{1}{\left|I_{\sigma}^{k}\right|} \int_{I_{\sigma}^{k}} w .
$$

Finally, from (13)(ii) we have

$$
\frac{1}{\left|J^{\prime}\right|} \int_{J^{\prime}} w \leqslant \frac{2}{\left|J^{k_{n}}\right|} \int_{J^{k_{n}}} w \leqslant 2 B \underset{J^{k_{n}}}{\operatorname{essinf}} w \leqslant \frac{2 B}{\left|I_{s}^{k_{n}}\right|} \int_{I_{s}^{k_{n}}} w
$$

and, combining this with the two previous inequalities, we obtain (15). From (15) and the assumption $M w \leqslant B w$ we obtain that for $k_{n} \leqslant l<k_{n+1}$

$$
\bigcup_{j:(l, j) \in P_{l}} I_{j}^{l} \subset\left\{w>\frac{3^{\left(l-k_{n}\right) \delta}\left|J^{\prime}\right|_{w}}{2 B^{2}\left|J^{l}\right|}\right\} \cap J^{l} .
$$


Since $w$ satisfies the $A_{\infty}$ condition [3], there are positive constants $C, \eta$ such that $|E|_{w} /|I|_{w} \leqslant C(|E| /|I|)^{\eta}$ whenever $E \subset$ an interval $I$. Taking for $E$ the set on the right side of (16), we conclude from the $A_{\infty}$ condition and the inequality

$$
\left|\{w>\lambda\} \cap J^{\prime}\right| \leqslant \lambda^{-1}\left|J^{\prime}\right|_{w},
$$

that $|E|_{w}$ is dominated by $\left|J^{l}\right|_{w}$ times $C\left(2 B^{2} / 3^{\left(l-k_{n}\right) \delta}\right)^{\eta}$. It follows that the left side of (14) is dominated by $\sum_{l=k_{n}}^{\infty} C 3^{\left(k_{n}-l\right) \delta \eta} \leqslant C$, as required.

We can now complete the proof. We have

$$
\begin{aligned}
h(x) & =\sum_{(k, j) \in P} \frac{\left|I_{j}^{k}\right|_{w}}{\left|J_{i}^{k}\right|_{w}}\left(\frac{1}{\left|J_{i}^{k}\right|} \int_{J_{i}^{k}} w\right) \chi_{J_{i}^{k}}(x) \\
& =\sum_{n} \sum_{\substack{l \in G \\
k_{n} \leqslant l<k_{n+1}}}\left(\sum_{(l, j) \in P_{l}} \frac{\left|I_{j}^{l}\right|_{w}}{\left|J^{l}\right|_{w}}\right)\left(\frac{1}{|J|^{l}} \int_{J^{\prime}} w\right) \\
& \leqslant \sum_{n} 2 C\left(\frac{1}{\mid J^{k_{n} \mid}} \int_{J^{k_{n}}} w\right)
\end{aligned}
$$

by (13)(ii) and (14). By (13)(i), this last sum is dominated by twice its largest term which in turn is dominated by $C M w(x) \leqslant C B w(x)$. Thus $h(x) \leqslant C w(x)$ and, combining this with (10) and (12), we obtain (7). Letting $N \downarrow-\infty$ and using (6), we obtain (4).

\section{REFERENCES}

1. K. Andersen and B. Muckenhoupt, Weighted weak type Hardy inequalities with applications to Hilbert transforms and maximal functions, Studia Math. 72 (1982), 9-26.

2. M. Christ and R. Fefferman, A note on weighted norm inequalities for the Hardy-Littlewood maximal operator, Proc. Amer. Math. Soc. 87 (1983), 447-448.

3. R. Coifman and C. Fefferman, Weighted norm inequalities for maximal functions and singular integrals, Studia Math. 51 (1974), 241-250.

4. R. Coifman, P. Jones and J. Rubio de Francia, Constructive decomposition of BMO functions and factorization of $A_{p}$ weights, Proc. Amer. Math. Soc. 87 (1983), 675-676.

5. R. Hunt, D. Kurtz and C. Neugebauer, $A$ note on the equivalence of $A_{p}$ and Sawyer's condition for equal weights, Proc. Conf. on Harmonic Analysis in Honor of Antoni Zygmund (1981: Chicago, Ill.), Vol. 1, Wadsworth Math. Ser., 1983, pp. 156-158.

6. B. Jawerth, Weighted inequalities for maximal operators: linearization, localization and factorization, preprint.

7. P. Jones, Factorization of $A_{p}$ weights, Ann. of Math. (2) 111 (1980), 511-530.

8. B. Muckenhoupt, Weighted norm inequalities for the Hardy maximal function, Trans. Amer. Math. Soc. 165 (1972), 207-226.

9. B. Muckenhoupt and R. L. Wheeden, Some weighted weak-type inequalities for the Hardy-Littlewood maximal function and the Hilbert transform, Indiana Univ. Math. J. 26 (1977), 801-816.

10. E. Sawyer, Two weight norm inequalities for certain maximal and integral operators, Proc. Conf. on Harmonic Analysis (Minneapolis, 1981), Lecture Notes in Math., Vol. 908, Springer-Verlag, Berlin and New York, 1982, pp. 102-127.

11. E. M. Stein and G. Weiss, Interpolation of operators with change of measures, Trans. Amer. Math. Soc. 87 (1958), 159-172.

Department of Mathematics, McMaster University, Hamilton L8S 4K1, Ontario, Canada 